\title{
A Possible New Sequence of DIBs
}

\author{
A. Bondar ${ }^{1}$ and J. Krełowski ${ }^{2}$ \\ ${ }^{1}$ IC AMER, National Academy of Sciences of Ukraine \\ Akademika Zabolotnoho Str 27, 03680, Kyiv, Ukraine \\ email: arctur@icamer.org.ua \\ ${ }^{2}$ Toruń Center for Astronomy, Nicholas Copernicus University, \\ Gagarina 11, Pl-87-100, Toruń, Poland \\ email: jacek@astri.uni.torun.pl
}

\begin{abstract}
One of the key factors in explaining nature of DIB carriers is to find regularities in their spectrum. Such a regular set of DIBs exists within interval $\lambda \lambda 6770-6865 \AA$. A possible new sequence of weak DIBs between $\lambda \lambda 5910-5990 \AA$ is presented here. The new sequence is clearly visible in high resolution spectra $(\mathrm{R} \gtrsim 100,000)$ of some hot, reddened stars. Wavenumbers' differences, or spacings, between the most strong bands have values $\sim 35, \sim 40 \mathrm{~cm}^{-1}$, which are very similar to those, found for DIBs near $6800 \AA$.
\end{abstract}

Keywords. ISM: general, ISM: lines and bands, ISM: molecules

\section{Overview}

The new sequence is definitely detectable in spectra of several stars included in survey by Bondar (2012). It is demonstrated in Fig. 1. There are spectra obtained with two spectrographs: UVES and HARPS. The groups of the most strong bands are marked with arrows and their mean central wavelengths are pointed.

Fig. 2 depicts "updated" sequence of DIBs in the range $\lambda \lambda 6770-6865 \AA$ introduced in work by Herbig (1988). There are central wavelengths of DIBs (bottom) and wavenumbers' scale with spacings between the most strong bands (top) presented. Several weak features (their wavelengths are marked with "1"), were not considered by G. Herbig as members of this sequence. But their inclusion in the sequence allows to form another combinations of DIBs and to find new spacings. Mean central wavelengths of the bands were taken from survey by Bondar (2012).

Three weak bands were added from work by Hobbs et al. (2008). Their wavelengths are noted with "2" and positions in the spectrum are marked with arrows.

Wavenumbers were calculated for atmospheric conditions of La Silla observatory assuming that energies of ground levels for DIBs' carriers are close to zero in interstellar medium.

As a result many repeating spacings can be traced: $\sim 40, \sim 35, \sim 21, \sim 19 \mathrm{~cm}^{-1}$ etc. Moreover, in some cases, spacings $\sim 40 \mathrm{~cm}^{-1}$ can be divided into smaller, two ones, with values close to 19 and $21 \mathrm{~cm}^{-1}$ (e.g. 40.60 into 19.35 and $21.25 \mathrm{~cm}^{-1}, 40.32$ into 19.33 and $20.99 \mathrm{~cm}^{-1}$ etc.). The spacings, noted with "3" were described in work by Herbig (1988).

The new sequence of DIBs, including the other bands (not all marked) between 5908$6015 \AA$, is presented on Fig. 3. A few bands beyond $5990 \AA$ were also included to get more different values of spacings. Found repeating differences are: $\sim 40-41, \sim 34-37, \sim 30$ $32 \mathrm{~cm}^{-1}$ etc. There are also shown spacings between close pairs ("doublets"), ranging from $1.89 \mathrm{~cm}^{-1}$ to $5.41 \mathrm{~cm}^{-1}$. 


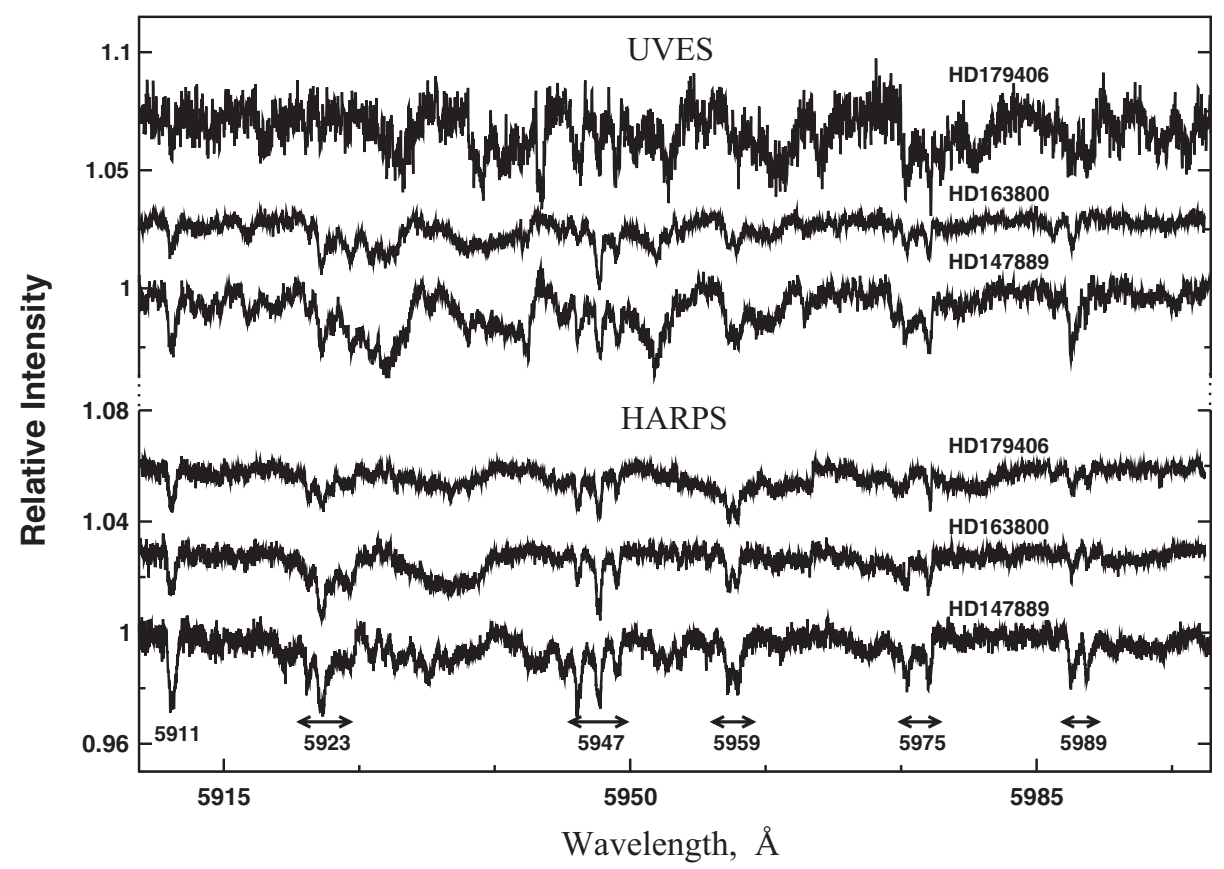

Figure 1. The possible new sequence of DIBs between 5910 and $5990 \AA$.

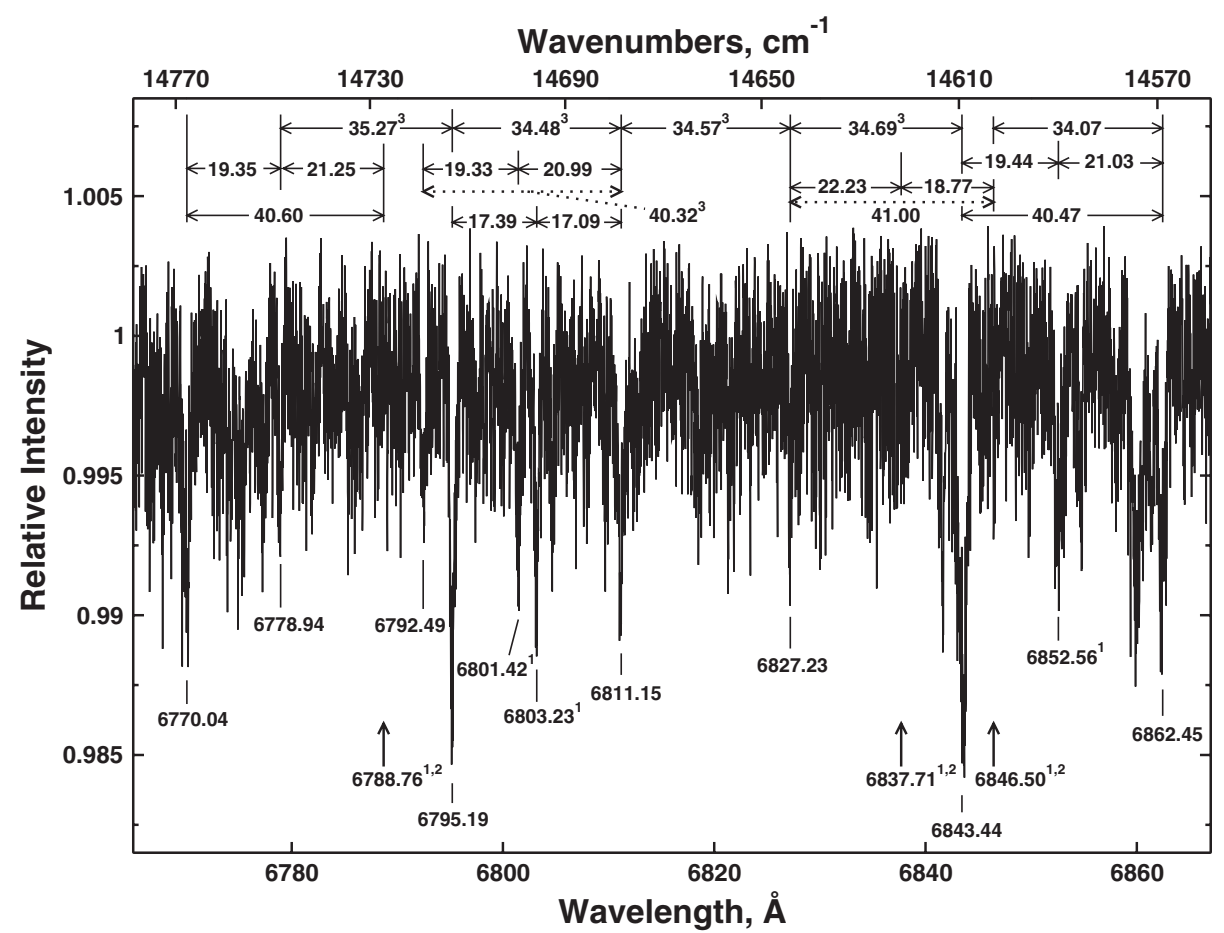

Figure 2. The sequence of DIBs in the range 6770-6865 $\AA$. Combined HARPS spectrum of HD163800 and 20 Aql. 


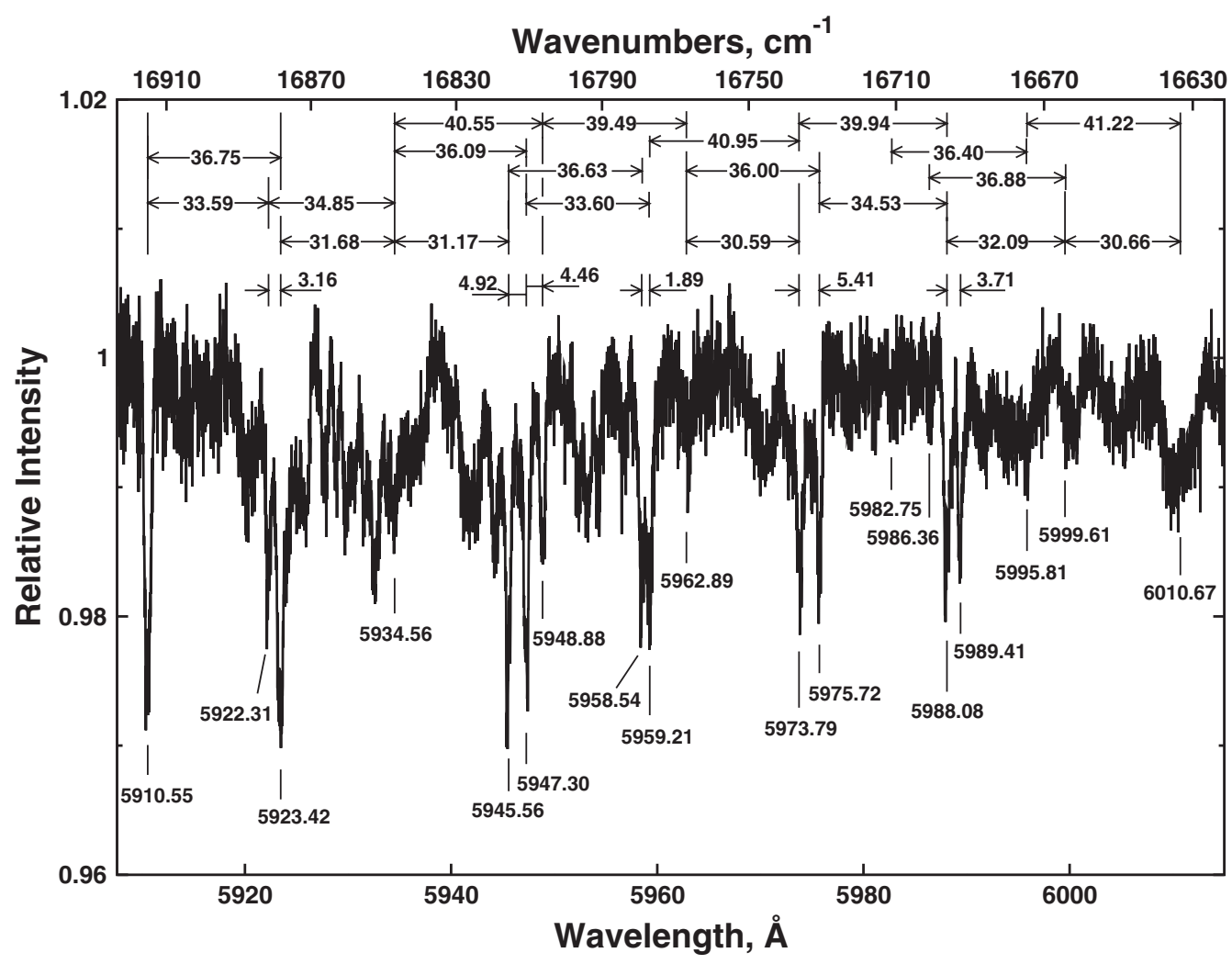

Figure 3. The new sequence of DIBs. HARPS spectrum of HD147889.

\section{Summary and prospects}

The spacings between some DIBs in the new sequence, such as $\sim 40-41, \sim 34-37 \mathrm{~cm}^{-1}$ are similar to those for bands from the spectral range $6770-6865 \AA$. It was showed in paper by Herbig (1988), that such values were too small for explaining either vibrational and rotational structures or rotation-vibration transitions in spectra of some diatomic molecules. On the other hand, spacings with $\sim 32$ and $\sim 34 \mathrm{~cm}^{-1}$ are close to energies of vibrational modes of large molecules, which may cause vibronic progressions in DIBs' spectra. Such progressions were analyzed in work by Duley \& Kuzmin (2010).

Despite these interesting findings we should keep in mind that coincidences in values of spacings can be coincidental because of the great number of bands in the considered range of the spectrum (this is probably the case for the spacings formed with the relatively broad band at $6010.67 \AA$; see Fig. 3). Thus consistency of features in the new sequence needs to be verified.

Obtaining spectra of high resolution with high $\mathrm{S} / \mathrm{N}$ ratio will allow to reveal more new weak spectral features, get detailed view of the above considered ranges of spectrum and to measure wavelengths (as well as wavenumbers) with higher precision.

\section{References}

Herbig, G. H. 1988, ApJ, 331, 999

Hobbs, L. M., York, D. G., Snow, T. P., Oka, T., Thorburn, J. A., Bishof, M., Friedman, S. D., McCall, B. J., Rachford, B., Sonnentrucker, P., \& Welty, D. E. 2008, ApJ, 680, 1256

Duley, W. W. \& Kuzmin, S. 2010, ApJL, 712, 165

Bondar, A. V. 2012, MNRAS, 423, 725 